\title{
Après le « non » irlandais
}

\section{Dominique Rivière}

\section{(QpenEdition \\ Journals}

\section{Édition électronique}

URL : http://journals.openedition.org/echogeo/6453

DOI : 10.4000/echogeo.6453

ISSN : 1963-1197

\section{Éditeur}

Pôle de recherche pour l'organisation et la diffusion de l'information géographique (CNRS UMR 8586)

\section{Référence électronique}

Dominique Rivière, «Après le «non » irlandais », EchoGéo [En ligne], Sur le Vif, mis en ligne le 09 juillet 2008, consulté le 03 mai 2019. URL : http://journals.openedition.org/echogeo/6453 ; DOI : 10.4000/ echogeo.6453

Ce document a été généré automatiquement le 3 mai 2019.

\section{(c) (i) (9)}

EchoGéo est mis à disposition selon les termes de la licence Creative Commons Attribution - Pas d'Utilisation Commerciale - Pas de Modification 4.0 International 


\title{
Après le « non » irlandais
}

\author{
Dominique Rivière
}

1 L'Irlande, $19^{\mathrm{e}}$ pays à voter sur la ratification du Traité de Lisbonne, était aussi le seul devant le faire par voie référendaire. Comme on le sait, il l'a rejeté à 53,4 \% des voix le 12 juin 2008. Or l'unanimité est requise pour que le texte entre en vigueur. Les 862000 votes « non » exprimés en Irlande ont ainsi renvoyé dans l'ornière un Traité péniblement relancé après le non français et néerlandais de 2005.

Disproportion des chiffres entre les 4 millions d'Irlandais et les quelque 500 millions d'Européens qui sont au final concernés par leur décision ? Certes, mais outre que celle-ci renvoie au primat des Etats dans la construction européenne, qui est sanctionné par le Traité de Lisbonne lui-même ${ }^{1}$, sur le fond, le vote irlandais reflète sans doute aussi le fait que le Traité n'a pas su recréer d'« élan européen ». En ce sens, quelque soit le choix qui sera adopté par Conseil européen à la mi-octobre 2008, il ne peut être considéré comme un simple accident de parcours.

\section{Divorce entre opinion publique et projet européen}

3 Tout d'abord, si le non irlandais a une dimension inquiétante, c'est précisément parce qu'il émane d'un pays qui, sans être parmi les plus europhiles, ne témoigne pas non plus d'un euro scepticisme structurel, comme c'est le cas par exemple de son voisin le Royaume-Uni. $80 \%$ des Irlandais qui ont voté non se disent favorables au maintien de l'Irlande dans l'Union européenne (Sondage Eurobaromètre, juin 2008); par ailleurs très peu d'entre eux estiment que l'Union européenne telle qu'elle est "n'a pas besoin d'amélioration» (tableau 1) : ce n'est donc pas par indifférence qu'ils ont exprimé leur désaccord avec le Traité. En outre, même s'il faut être prudent lorsqu'on associe l'essor économique de l'Irlande à son intégration communautaire - ses liens avec le monde anglo-saxon et en particulier les Etats-Unis ont joué aussi un rôle -, il est patent que les Irlandais ont bénéficié de l'Union européenne ${ }^{2}$, que ce soit sa politique régionale, puisque le pays a bénéficié de l'Objectif 1 de cette politique ainsi que du fonds de cohésion, ou dans ses aspects plus généraux. En particulier, le "modèle irlandais " s'est en partie fondé sur le jeu d'avantages fiscaux au sein du Marché unique - que les Irlandais 
craignent de perdre (tableau 1). En somme, l'Irlande est un pays plutôt bienveillant à l'égard de la construction européenne. Toutefois, ce n'est pas la première fois qu'elle vote non à un texte européen : elle l'a déjà fait à propos du Traité de Nice. Mais l'écho du non de 2008 est plus important car il joue dans un contexte général marqué par les doutes sur le projet européen et le réveil des égoïsmes nationaux.

4 Par ailleurs, selon les différents sondages effectués auprès des Irlandais, que ce soit avant ou après le vote, la principale raison de voter non a été le fait que les personnes interrogées «ne savent pas sur quoi elles votent » (tableau 1). La complexité et la longueur du texte ont donc joué contre lui, comme a joué sans doute la situation confuse dans laquelle se trouve d'une façon générale le projet européen sur le plan de la communication, en Irlande comme ailleurs. Comme le relève le journaliste français Jean Quatremer, en matière de questions européennes on est entré aujourd'hui dans l' " ère de la rumeur ", et il s'avère bien difficile de démêler effets d'annonce et réalités, attentes et craintes supposées ou réelles des citoyens européens ${ }^{3}$, voire tout simplement de communiquer sur les décisions ou les projets émanant de l'Union européenne, tant les opinions se sont durcies dans un sens ou dans un autre depuis 2005.

Tableau 1 - Les principales raisons de voter « non » au Traité de Lisbonne

\begin{tabular}{|c|c|}
\hline Motifs & $\%$ \\
\hline « parce que je n'en sais pas assez sur le traité... » & 22 \\
\hline "pour protéger l'identité irlandaise » & 12 \\
\hline "pour sauvegarder la neutralité irlandaise en matière de sécurité et de défense » & 6 \\
\hline « je n'ai pas confiance dans nos hommes politiques » & 6 \\
\hline «nous allons perdre le droit d'avoir un Commissaire dans chaque commission » & 6 \\
\hline "pour protéger notre régime fiscal » & 6 \\
\hline « je suis contre l'idée d'une Europe unie » & 5 \\
\hline «pour protester contre la politique du gouvernement » & 4 \\
\hline "pour éviter que l'Europe parle d'une seule voix sur des sujets globaux » & 4 \\
\hline " parce que les grands États membres décident dans les affaires européennes » & 4 \\
\hline "pour protéger l'influence des petits États» & 3 \\
\hline
\end{tabular}




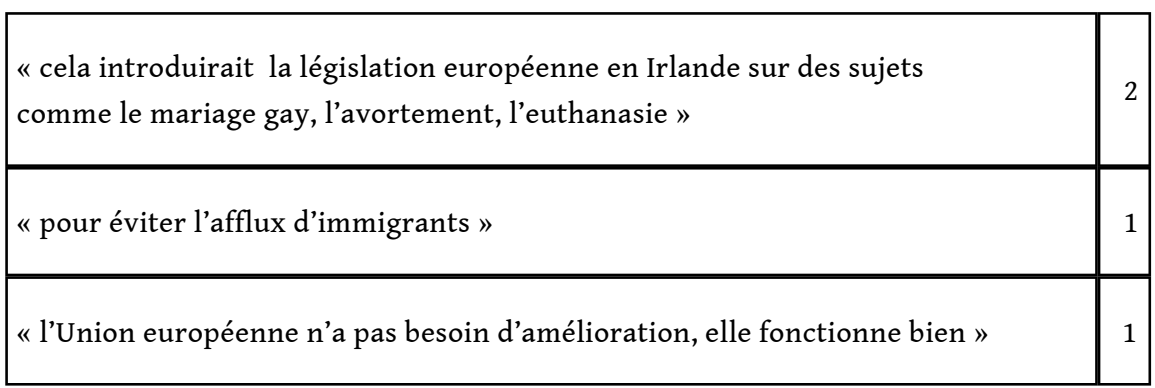

Source : sondage effectué en Irlande, Eurobaromètre n²45, juin 2008.

5 Quoi qu'il en soit, et sans revenir sur le contexte général européen évoqué plus haut, la plupart des commentateurs sont d'accord pour dire que le divorce est ici patent entre opinion publique et élite politique : la classe politique irlandaise avait fait massivement campagne pour le oui, or le désavœu de la classe politique précisément arrive au quatrième rang des motifs du non (tableau 1). Le fait que les questions européennes n'aient pas le monopole de l'expression de ce divorce ne rend pas les choses moins inquiétantes : sans préjuger du résultat si d'autres référendums avaient été effectués en 2008 en Union européenne, le non irlandais éveille d'évidents échos avec d'autres non, et en particulier le non français de 2005. Le divorce est patent aussi entre les préoccupations actuelles des Européens (l'emploi, la vie chère, le coût de l'énergie, etc.) et les problématiques institutionnelles qui, si utiles soient-elles à la résolution des dits problèmes, semblent lointaines à la plupart d'entre eux. Fait significatif, le non a enregistré ses scores maximaux dans des contrées périphériques comme le Donegal, mais Cork et Dublin ont aussi été très partagées.

\section{Poursuivre la ratification: « voix de la raison » ou « acharnement thérapeutique »?}

D'emblée, le non irlandais a suscité une réaction politique bien différente du non français et néerlandais de 2005. Alors que celui-ci avait immédiatement signé le coup d'arrêt de la Constitution, le gouvernement Blair annonçant le gel du processus de ratification, rien de tel ne s'est produit en 2008. Quelques jours à peine après le vote irlandais, la Chambre des Lords britannique ratifiait le Traité - un sujet britannique a cependant fait appel ${ }^{4}$ - et le Conseil européen du 19 et 20 juin a donc pu constater que « les parlements de 19 Etats membres (ont) ratifié le traité et le processus de ratification se (poursuit) dans les autres Etats membres ", formulation prudente destinée à ne pas braquer quelques eurosceptiques notoires comme le chef du gouvernement tchèque, qui doit prendre la Présidence de l'Union en janvier 2009. Peine perdue? Le $1^{\text {er }}$ juillet, alors même que la France - qui, par la voix de Nicolas Sarkozy, prône comme on le sait la poursuite de la ratification - prenait elle-même cette fonction, le Président de la République polonaise annonçait son intention de ne pas ratifier le Traité, pourtant voté par la Diète en avril! Son Premier ministre, le libéral Donald Tusk, a immédiatement tempéré ces propos. Deux jours plus tard, le Parlement de Chypre approuvait quant à lui le Traité, et l'Espagne, qui avait prévu initialement d'achever la ratification de Lisbonne à l'automne prochain, a décidé d'accélérer le mouvement et de le faire courant juillet... Entre chaud et froid et d'un rebondissement à un autre, on a parfois l'impression que la ratification du Traité de Lisbonne commence à ressembler à un mauvais vaudeville. 
7 Sur le fond, poursuivre la ratification, est-ce suivre la « voix de la raison » ou cela relève-til d'un « acharnement thérapeutique » ((Jean-Louis Bourlanges, ex-député européen UDF sur LCI, le 13-6-08) ? Sans préjuger de la décision finale, qui devrait être prise à la mioctobre 2008, il semble, en simplifiant le débat public tel qu'il s'expose dans la presse, que trois issues se présentent :

- « aller de l'avant » en considérant le vote irlandais comme un incident surmontable : dans cette hypothèse, c'est aux Irlandais de trouver une solution acceptable, qui passerait en l'occurrence par un second vote de leur part (comme ils l'avaient fait pour Nice). Cette solution est pour l'heure la plus probable si l'on en croit les prises de position des divers chefs d'État et la dynamique enclenchée. En outre, si l'on considère les enquêtes d'opinion, elle semble moins compliquée à monter que l'on n'aurait pu le craindre car en Irlande même, les trois-quarts de ceux qui ont voté non estiment que la situation qui s'est créée va permettre au gouvernement irlandais de renégocier «quelques exceptions » en faveur de leur pays (38\% seulement de ceux qui ont voté oui partagent cet espoir). Toutefois, une telle manœuvre, si c'est le choix qui ressort des propositions du gouvernement irlandais et des décisions du Conseil européen d'octobre $2008^{5}$, ne résoudra pas sur le fond le divorce entre les opinions publiques européennes et les modalités de la ratification. Au-delà du cas irlandais, elle pourrait avoir un effet boomerang sur la participation des électeurs aux prochaines élections du Parlement européen, prévues en juin 2009. Elle risque aussi d'ouvrir la boîte de Pandore des revendications spécifiques à tel ou tel pays.

- « continuer sans l'Irlande » : cette hypothèse - difficile à défendre sur le plan juridique n'est pas seulement rejetée par les Irlandais eux-mêmes, qu'ils aient voté oui ou non, mais aussi par la plupart des autres États européens, tout particulièrement les plus petits d'entre eux, en particulier par la voix de Jean-Claude Juncker ${ }^{6}$. Et pour cause : parmi les motifs récurrents du non irlandais, on relève en effet différents arguments touchant à la question du poids des petits États dans une Union européenne élargie (tableau 1 : la perte d'un Commissaire, la crainte que l'Union européenne " parle d'une seule voix », la crainte des grands États, la protection de l'influence des petits États). À cet égard, il est évident qu'isoler l'Irlande serait perçu comme une punition, et aurait des effets délétères sur l'ensemble du projet européen.

- « abandonner Lisbonne et adopter Nice » : cette hypothèse, défendue par des personnalités comme Hubert Védrine, ex-Ministre des affaires étrangères français, est pour l'heure minoritaire tant le Traité de Nice de 2001 alimente les craintes de paralysie du projet européen. Elle plaide toutefois pour l'acceptation de la défaite du projet global qu'a été d'abord le Traité constitutionnel puis aujourd'hui le Traité de Lisbonne, ce qui implique donc de revenir précisément à l'application du Traité de Nice. En somme, il s'agit ici de fonder la poursuite du projet européen sur des bases certes plus étroites mais qui ont l'avantage d'avoir été légitimées... au moins par le temps. Le corollaire de cette hypothèse est la promotion de l'idée d'une coopération renforcée entre « les États qui veulent aller de l'avant ", idée présente depuis le Traité d'Amsterdam et facilitée par le traité de Nice : celuici autorise la mise en oeuvre de telles coopérations dès lors que huit États se sont mis d'accord et "lorsqu'il a été établi au sein du Conseil que les objectifs qui leur sont assignés ne peuvent être atteints, dans un délai raisonnable, en s'en tenant aux dispositions pertinentes des traités".

8 En somme, ces trois solutions comportent des risques. Pour la première, c'est celui d'écorner encore davantage la légitimité politique du projet européen, au nom de l'efficacité immédiate (clore le processus) et à moyen terme (Lisbonne est «meilleur que Nice»). Pour la seconde, et dans une moindre mesure pour la troisième, le risque est celui 
de sacrifier le principe de l'intégration au profit de celui d'une Union européenne à géométrie variable. Quant à une autre hypothèse, qui aurait été la refonte du texte (le fameux Plan B de 2005), elle n'est pas du tout à l'ordre du jour.

\section{BIBLIOGRAPHIE}

Eurobaromètre Flash n²45, 18 juin 2008 (numéro spécial sur l'Irlande)

Commission européenne, 2001, Unité de l'Europe, solidarité des peuples, diversité des territoires, deuxième rapport sur la cohésion économique et sociale, Office des publications officielles des CE Commission européenne, 2007, Growing regions, growing Europe (quatrième rapport sur la cohésion économique et sociale), Office des publications officielles des CE

ESPON (European spatial planning observation network), 2006, Territory matters for competitiveness and cohesion, ESPON synthesis report III

Didaoui K, 2007, Traité modificatif, explication de texte, Confrontation Europe, oct.-déc, p 6-9

Fondation Robert Schuman, 2007, Le Traité de Lisbonne expliqué en dix fiches, www.robert-schuman.eu

Sénat français, 2007, Le traité de Lisbonne,http://www.senat.fr/rap/r07-188/r07-1883.html

Ricard-Nihoul G, 2007, Le Traité de Lisbonne : relance d'un traité ou traité de la relance ?, Notre

Europe, nov 2007, p 1-10

- Sites utiles

www.Europa.eu.int

www.senat.fr

www.traite-de-lisbonne.fr

www.robert-schuman.eu

- Textes précédant le Traité de Lisbonne

- Traité de Nice, JO des Communautés européennes, 10-3-12001

Pour un complément d'information sur le Traité de Nice et la coopération renforcée, on peut consulter utilement les sites : http://europa.eu/scadplus/nice_treaty/cooperations_fr.htm et http://www.senat.fr/rap/ro0-202/r00-2024.html

- Traité d'Amsterdam, JO des Communautés européennes, 10-11-1997

- Projet de Traité établissant une constitution pour l'Europe, 3-2005

\section{NOTES}

1. Le Traité de Lisbonne remet-il l'Europe «sur les rails »? 
2. Ceux qui ont voté oui donnent comme premier motif : "c'était l'intérêt de l'Irlande » (32\%) suivi de «l'Irlande a bénéficié de l'Union européenne » (19\%), Eurobaromètre $n^{\circ} 245$, juin 2008. C'est dans les années 1990, alors qu'elle était pleinement éligible à l'objectif 1, que l'Irlande a le plus bénéficié des fonds structurels. Selon les estimations communautaires (Commission européenne, 2001 ) leur apport serait de l'ordre du 2,2\% du PIB irlandais en 1989, 3,7\% en 1999. A titre comparatif, pour l'Espagne, souvent considérée -jusqu'à la récente crise immobilièrecomme l'autre «bon élève » de l'Union européenne, cet apport des fonds structurels est estimé à respectivement $0,8 \%$ et $3,1 \%$ du PIB. À partir du programme 2000-2006, l'Irlande a perdu progressivement ce statut prioritaire, du fait même du haut niveau de développement acquis par le pays : son PIB par habitant est à l'indice 141,4 si on prend l'Union européenne à 27 comme indice 100 (Commission européenne, 2007, données 2004 exprimées en standard de pouvoir d'achat). Pour un bilan global des dépenses des fonds structurels et de pré-adhésion dans les années 1990, voir aussi ESPON 2006, carte 14 p 73.

3. Par exemple, la crainte de l'immixtion de l'Union européenne dans des sujets "sociétaux » comme l'avortement, le mariage gay, l'euthanasie, a souvent été évoquée dans les semaines précédant le vote, alors qu'elle semble en fait avoir peu pesé sur le vote non si l'on en juge d'après le sondage Eurobaromètre 2008. Mais "protéger l'identité irlandaise " est une préoccupation plus diffuse.

4. En conséquence, un juge de la Haute Cour de Londres a demandé le report de la ratification définitive tant qu'il ne se sera pas prononcé sur un recours exigeant un référendum sur la question dans.

5. Il pourrait s'agir -sous réserve - d'un nouveau vote sur un texte qui resterait inchangé mais serait assorti d'une déclaration garantissant aux Irlandais par exemple leur neutralité, leur législation en matière d'avortement ou encore l'absence d'interférence de l'Union européenne dans la fiscalité.

6. Le Premier ministre luxembourgeois, qui est par ailleurs un des candidats favoris au poste de Président du Conseil européen déclare ainsi peu après le non irlandais : "Je ne suis pas d'accord avec ceux qui veulent à tout prix isoler l'Irlande, la mettre sur le banc des accusés et l'obliger à choisir entre le traité de Lisbonne et la sortie de l'Union. Jamais on n'aurait dit ça à la France, donc on ne peut pas le dire à l'Irlande », dans Libération, 19/06/2008.

\section{RÉSUMÉS}

Le Traité de Lisbonne a été rejeté par référendum en Irlande le 12 juin 2008. Loin d'être un incident de parcours, le vote irlandais est symptomatique d'un divorce persistant entre opinions publiques et projet européen.Parmi les issues possibles, la plus probables est néanmoins celle de la poursuite de la ratification qui serait assortie d'un nouveau vote irlandais.

The Treaty of Lisbon has been rejected by the referendum of the 12 of june 2008. Far away from being a mere «incident ", this votation is symptomatic of a persistent "divorce " between the public opinions and the european project. Nevertheless, the most probale issue is the proceeding of the ratification process which would be accompanied with a new vote in Irland. 
INDEX

Keywords : crisis, European construction, Irland, treaty of Lisbon

Mots-clés : construction européenne, crise, Irlande, traité de Lisbonne

\section{AUTEUR}

\section{DOMINIQUE RIVIÈRE}

Dominique Rivière (rivieredomi@aliceadsl.fr) est professeur de géographie à l'Université ParisDiderot et membre de l'UMR Géographie-Cités.Parmi ses publications, nous pouvons citer :

- 2005, 2006, 2007, 2008, L'Europe, dans Dossiers et images économiques du Monde, Paris, A Colin. - 2004, L'Italie des régions à l'Europe, Paris, A Colin, 252 p. 